\title{
The Hyper Suprime-Cam SSP Survey: Overview and Survey Design
}

Hiroaki Aihara ${ }^{1}$, Nobuo Arimoto ${ }^{2,3}$, Robert Armstrong ${ }^{4}$,

Stéphane Arnouts ${ }^{5}$, Neta A. Bahcall ${ }^{4}$, Steven Bickerton ${ }^{6}$, James Bosch ${ }^{4}$, Kevin Bundy ${ }^{7,8}$, Peter L. Capak ${ }^{9}$, James H. H. Chan ${ }^{10,11}$, Masashi Chiba ${ }^{12}$, Jean Coupon ${ }^{13}$, Eiichi Egami ${ }^{14}$, Motohiro Enoki ${ }^{15}$, Francois Finet ${ }^{3}$, Hiroki Fujimori ${ }^{16}$, Seiji Fujimoto ${ }^{17}$, Hisanori Furusawa ${ }^{18}$, Junko Furusawa ${ }^{18}$, Tomotsugu Goto ${ }^{19}$, Andy Goulding ${ }^{4}$, Johnny P. Greco ${ }^{4}$, Jenny E. Greene ${ }^{4}$, James E. Gunn ${ }^{4}$, Takashi Hamana ${ }^{18}$, Yuichi Harikane $e^{1,17}$, Yasuhiro Hashimoto ${ }^{21}$, Takashi Hattori ${ }^{3}$, Masao Hayashi ${ }^{18}$, Yusuke Hayashi ${ }^{18}$, Krzysztof G. Hełminiak ${ }^{22}$, Ryo Higuchi ${ }^{1,17}$, Chiaki Hikage ${ }^{7}$, Paul T. P. Ho ${ }^{10,23}$, Bau-Ching Hsieh ${ }^{10}$, Kuiyun Huang ${ }^{24}$, Song Huang ${ }^{8,7}$, Hiroyuki Ikeda ${ }^{18}$, Masatoshi Imanishi ${ }^{18,2}$, Akio K. Inoue ${ }^{25}$, Kazushi Iwasawa ${ }^{26,27}$, Ikuru Iwata ${ }^{3,2}$, Anton T. Jaelani ${ }^{12}$, Hung-Yu Jian ${ }^{10}$, Yukiko Kamata ${ }^{18}$, Hiroshi Karoji ${ }^{28,4}$, Nobunari Kashikawa ${ }^{18,2}$, Nobuhiko Katayama ${ }^{7}$, Satoshi Kawanomoto ${ }^{18}$, Issha Kayo ${ }^{29}$, Jin Koda ${ }^{30}$, Michitaro Koike ${ }^{18}$, Takashi Kojima ${ }^{1,17}$, Yutaka Komiyama ${ }^{18,2}$, Akira Konno ${ }^{17}$, Shintaro Koshida ${ }^{3}$, Yusei Koyama ${ }^{3,2}$, Haruka Kusakabe ${ }^{20}$, Alexie Leauthaud ${ }^{7,8}$, C.-H. Lee ${ }^{3}$, Lihwai Lin ${ }^{10}$, Yen-Ting Lin ${ }^{10}$, Robert H. Lupton ${ }^{4}$, Rachel Mandelbaum ${ }^{31}$, Yoshiki Matsuoka ${ }^{18,32}$, Elinor Medezinski ${ }^{4}$, Sogo Mineo ${ }^{18}$, Shoken Miyama ${ }^{33,34}$, Hironao Miyatake ${ }^{35,7}$, Satoshi Miyazaki ${ }^{18,2}$, Rieko Momose ${ }^{19}$, Anupreeta More ${ }^{7}$, Surhud More ${ }^{7}$, Yuki Moritani ${ }^{7}$, Takashi J. Moriya ${ }^{18}$, Tomoki Morokuma ${ }^{36,7}$, Shiro Mukae ${ }^{17}$, Ryoma Murata ${ }^{7,1}$, Hitoshi Murayama ${ }^{7,37,38}$, Tohru Nagao $^{32}$, Fumiaki Nakata ${ }^{3}$, Mana Niida ${ }^{39}$, Hiroko Niikura ${ }^{1,7}$, Atsushi J. Nishizawa ${ }^{40}$, Yoshiyuki Obuchi ${ }^{18}$, Masamune Oguri ${ }^{41,7,1}$, Yukie Oishi ${ }^{18}$, Nobuhiro Okabe ${ }^{42,33,7}$, Sakurako Okamoto ${ }^{43}$, Yuki Okuraa ${ }^{44,45}$, Yoshiaki Ono ${ }^{17}$, Masato Onodera ${ }^{3}$, Masafusa Onoue ${ }^{18,2}$, Ken Osato ${ }^{1}$, Masami Ouchi ${ }^{17,7}$, Paul A. Price ${ }^{4}$, Tae-Soo Pyo ${ }^{3}$, Masao Sako ${ }^{46}$, Marcin Sawicki ${ }^{47}$, Takatoshi Shibuya ${ }^{17}$, Kazuhiro Shimasaku ${ }^{20,41}$, Atsushi Shimono ${ }^{7}$, Masato Shirasaki ${ }^{18}$, John D. Silverman ${ }^{7}$, Melanie Simet $^{48}$, Joshua Speagle ${ }^{49,7}$, David N. Spergel ${ }^{4,50}$, Michael A. Strauss ${ }^{4, *}$, Yuma Sugahara ${ }^{1,17}$, Naoshi Sugiyama ${ }^{51,7}$, Yasushi Suto ${ }^{1,41}$, Sherry H. Suyu ${ }^{10,52,53}$, Nao Suzuki ${ }^{7}$, Philip J. Tait ${ }^{3}$, Masahiro Takada ${ }^{7, *}$, Tadafumi Takata ${ }^{18,2}$, Naoyuki Tamura ${ }^{7}$, Manobu M. Tanaka ${ }^{54}$, Masaomi Tanaka ${ }^{18}$, Masayuki Tanaka ${ }^{18}$, Yoko Tanaka ${ }^{3}$, Tsuyoshi Terai ${ }^{3}$, Yuichi Terashima ${ }^{32}$, Yoshiki Toba ${ }^{10}$, Nozomu Tominaga ${ }^{55,7}$, Jun Toshikawa ${ }^{17}$, Edwin L. Turner ${ }^{4,7,1}$, 
Tomohisa Uchida $^{54}$, Hisakazu Uchiyama ${ }^{2}$, Keiichi Umetsu ${ }^{10}$,

Fumihiro Uraguchi ${ }^{18}$, Yuji Urata ${ }^{56}$, Tomonori Usuda ${ }^{18,2}$, Yousuke Utsumi ${ }^{33}$, Shiang-Yu Wang ${ }^{10}$, Wei-Hao Wang ${ }^{10}$, Kenneth C. Wong ${ }^{18,10}$, Kiyoto Yabe ${ }^{7}$, Yoshihiko Yamada ${ }^{18}$, Hitomi Yamanoi ${ }^{18}$, Naoki Yasuda ${ }^{7}$, Sherry Yeh ${ }^{3}$, Atsunori Yonehara ${ }^{57}$, Suraphong Yuma ${ }^{58}$

${ }^{1}$ Department of Physics, University of Tokyo, Tokyo 113-0033, Japan

${ }^{2}$ Department of Astronomy, School of Science, Graduate University for Advanced Studies (SOKENDAI), 2-21-1, Osawa, Mitaka, Tokyo 181-8588, Japan

${ }^{3}$ Subaru Telescope, National Astronomical Observatory of Japan, 650 N Aohoku PI, Hilo, HI 96720

${ }^{4}$ Department of Astrophysical Sciences, Princeton University, 4 Ivy Lane, Princeton, NJ 08544

${ }^{5}$ CNRS, Laboratoire d'Astrophysique de Marseille, UMR 7326, Aix Marseille Universit,

F-13388, Marseille, France

${ }^{6}$ Orbital Insight, 100 W. Evelyn Ave. Mountain View, CA 94041

${ }^{7}$ Kavli Institute for the Physics and Mathematics of the Universe (Kavli IPMU, WPI), UTIAS,

University of Tokyo, Chiba 277-8583, Japan

${ }^{8}$ Department of Astronomy and Astrophysics, University of California, Santa Cruz, 1156 High

Street, Santa Cruz, CA 95064 USA

${ }^{9}$ California Institute of Technology, 1200 E. California Blvd., Pasadena, CA, 91125, USA

${ }^{10}$ Academia Sinica Institute of Astronomy and Astrophysics, P.O. Box 23-141, Taipei 10617, Taiwan

${ }^{11}$ Department of Physics, National Taiwan University, 10617 Taipei, Taiwan

${ }^{12}$ Astronomical Institute, Tohoku University, 6-3, Aramaki, Aoba-ku, Sendai, Miyagi, 980-8578, Japan

${ }^{13}$ Department of Astronomy, University of Geneva, ch. d'Écogia 16, 1290 Versoix, Switzerland

${ }^{14}$ Steward Observatory, University of Arizona, 1540 East Second Street Tucson, AZ 85721-0064, USA

${ }^{15}$ Faculty of Business Administration, Tokyo Keizai University, Kokubunji, Tokyo, 185-8502, Japan

${ }^{16}$ MEISEI ELECTRIC CO., LTD, 2223 Naganuma, Isesaki, Gumma, Japan

17 Institute for Cosmic Ray Research, The University of Tokyo, 5-1-5 Kashiwanoha, Kashiwa, Chiba 277-8582, Japan

18 National Astronomical Observatory of Japan, 2-21-1 Osawa, Mitaka, Tokyo 181-8588, Japan

${ }^{19}$ Institute of Astronomy, National Tsing Hua University, 101, Section 2 Kuang-Fu Road, Hsinchu, Taiwan, 30013, R.O.C.

20 Department of Astronomy, Graduate School of Science, The University of Tokyo, 7-3-1 Hongo, Bunkyo, Tokyo, 113-0033, Japan

${ }^{21}$ Department of Earth Sciences, National Taiwan Normal University No.88, Sec. 4, Tingzhou Rd., Wenshan District, Taipei 11677, Taiwan

${ }^{22}$ Department of Astrophysics, Nicolaus Copernicus Astronomical Center, ul. Rabiańska 8, 87-100 Toruń, Poland

${ }^{23}$ East Asian Observatory, 660 N. A'ohoku Place, University Park, Hilo, Hawaii 96720, U.S.A.

${ }^{24}$ Department of Mathematics and Science, National Taiwan Normal University, Lin-kou District, New Taipei City 24449, Taiwan

${ }^{25}$ Department of Environmental Science and Technology, Faculty of Design Technology, Osaka Sangyo University, 3-1-1 Nakagaito, Daito, Osaka 574-8530, Japan

${ }^{26}$ Institut de Ciències del Cosmos (ICCUB), Universitat de Barcelona (IEEC-UB), Martí i Franquès, 1, 08028 Barcelona, Spain

${ }^{27}$ ICREA, Pg. Lluís Companys 23, 08010 Barcelona, Spain 
${ }^{28}$ National Institutes of Natural Sciences, 4-3-13 Toranomon, Minato-ku, Tokyo, JAPAN

29 Department of Liberal Arts, Tokyo University of Technology, Ota-ku, Tokyo 144-8650, Japan

${ }^{30}$ Department of Physics and Astronomy, Stony Brook University, Stony Brook, NY 11794-3800

${ }^{31}$ McWilliams Center for Cosmology, Department of Physics, Carnegie Mellon University, Pittsburgh, PA 15213, USA

${ }^{32}$ Research Center for Space and Cosmic Evolution, Ehime University, 2-5 Bunkyo-cho, Matsuyama, Ehime 790-8577, Japan

${ }^{33}$ Hiroshima Astrophysical Science Center, Hiroshima University, 1-3-1 Kagamiyama, Higashi-Hiroshima, Hiroshima, 739-8526, Japan

${ }^{34}$ Center for Planetary Science, Integrated Research Center of Kobe University, 7-1-48, Minamimachi, Minatojima, Chuo-ku, Kobe 650-0047, Japan

35 Jet Propulsion Laboratory, California Institute of Technology, Pasadena, CA 91109, USA

${ }^{36}$ Institute of Astronomy, University of Tokyo, 2-21-1 Osawa, Mitaka, Tokyo 181-0015,Japan

${ }^{37}$ Department of Physics and Center for Japanese Studies, University of California, Berkeley, CA 94720, USA

${ }^{38}$ Theoretical Physics Group, Lawrence Berkeley National Laboratory, MS 50A-5104, Berkeley, CA 94720

${ }^{39}$ Graduate School of Science and Engineering, Ehime University, 2-5 Bunkyo-cho, Matsuyama, Ehime 790-8577, Japan

${ }^{40}$ Institute for Advanced Research, Nagoya University Furocho, Chikusa-ku, Nagoya, 464-8602 Japan

41 Research Center for the Early Universe, University of Tokyo, Tokyo 113-0033, Japan

${ }^{42}$ Department of Physical Science, Hiroshima University, 1-3-1 Kagamiyama, Higashi-Hiroshima, Hiroshima 739-8526, Japan

${ }^{43}$ Shanghai Astronomical Observatory, 80 Nandan Rd., Shanghai 200030, China

44 RIKEN High Energy Astrophysics Laboratory, 2-1 Hirosawa, Wako, Saitama 351-0198, Japan

45 RIKEN BNL Research Center, BIdg. 510A, 20 Pennsylvania Street, Brookhaven National Laboratory, Upton, NY 11973

46 Department of Physics and Astronomy, University of Pennsylvania, 209 South 33rd Street, Philadelphia, PA 19104 USA

${ }^{47}$ Saint Mary's University, Department of Astronomy and Physics, 923 Robie Street, Halifax, NS B3H 3C3, Canada

48 University of California, Riverside, 900 University Avenue, Riverside, CA 92521, USA

49 Harvard University, 60 Garden St., Cambridge, MA 02138, USA

50 Center for Computational Astrophysics, Flatiron Institute, 162 5th Ave. New York, NY 10010

51 Department of Physics and Astrophysics, Nagoya University, Nagoya 464-8602, Japan

${ }^{52}$ Max-Planck-Institut für Astrophysik, Karl-Schwarzschild-Str. 1, 85748 Garching, Germany

${ }^{53}$ Physik-Department, Technische Universität München, James-Franck-Straße 1, 85748 Garching, Germany

54 Institute of Particle and Nuclear Studies, High Energy Accelerator Research Organization, 203-1 Shirakata, Tokai-mura, Naka-gun, Ibaraki, Japan, 319-1106

${ }^{55}$ Department of Physics, Faculty of Science, and Engineering, Konan University, 8-9-1 Okamoto, Kobe, Hyogo 658-8501, Japan

56 Institute of Astronomy, National Central University, Chung-Li 32054, Taiwan

57 Department of Astrophysics and Atmospheric Science, Faculty of Science, Kyoto Sangyo University, Motoyama, Kamigamo, Kita-ku, Kyoto, 603-8555, JAPAN

${ }^{58}$ Department of Physics, Faculty of Science, Mahidol University, Bangkok 10400, Thailand 


\section{${ }^{*}$ Corresponding Authors}

*E-mail: masahiro.takada@ipmu.jp, strauss@astro.princeton.edu

Received; Accepted

\section{Abstract}

Hyper Suprime-Cam (HSC) is a wide-field imaging camera on the prime focus of the $8.2 \mathrm{~m}$ Subaru telescope on the summit of Maunakea in Hawaii. A team of scientists from Japan, Taiwan and Princeton University is using HSC to carry out a 300-night multi-band imaging survey of the high-latitude sky. The survey includes three layers: the Wide layer will cover $1400 \mathrm{deg}^{2}$ in five broad bands (grizy), with a $5 \sigma$ point-source depth of $r \approx 26$. The Deep layer covers a total of $26 \mathrm{deg}^{2}$ in four fields, going roughly a magnitude fainter, while the UltraDeep layer goes almost a magnitude fainter still in two pointings of HSC (a total of $3.5 \mathrm{deg}^{2}$ ). Here we describe the instrument, the science goals of the survey, and the survey strategy and data processing. This paper serves as an introduction to a special issue of the Publications of the Astronomical Society of Japan, which includes a large number of technical and scientific papers describing results from the early phases of this survey.

Key words: TBD

\section{Introduction}

We live in a golden age for extragalactic astronomy and cosmology. We now have a quantitative and highly predictive model for the overall composition and expansion history of the Universe that is in accord with a large array of independent and complementary observations. Observations of galaxies over most of the 13.8 billion year history of the Universe have led to a broadbrush understanding of the basics of galaxy evolution. Studies of the structure of our Milky Way galaxy are in rough agreement with the current galaxy evolution paradigm. However, there are fundamental and inter-related questions that remain:

- What is the physical nature of dark matter and dark energy? Is dark energy truly necessary, or could the accelerated expansion of the Universe be explained by modifications of the law of gravity?

- How did galaxies assemble and how did their properties change over cosmic time? Can a coherent galaxy evolution model be found that fits both observations of the distant universe, as well as detailed studies of nearby galaxies including the Milky Way?

- What is the topology and timing of reionization of the intergalactic medium at high redshift? What were the sources of ultraviolet light responsible for that reionization?

This paper describes a comprehensive deep and wide-angle imaging survey of the sky designed to address these and other key questions in astronomy, using the Hyper Suprime-Cam (HSC), a wide-field imaging camera on the 8.2-meter Subaru telescope, operated by the National Astronomical Observatory of Japan (NAOJ) on the summit of Maunakea in Hawaii. The combination of the large aperture of the Subaru telescope, the large field of view (1.5 deg diameter) of HSC, and the excellent image quality of the site and the telescope make this a powerful instrument for addressing these fundamental questions in modern cosmology and astronomy. Under the Subaru Strategic Program (SSP), we began a survey using both broadand narrow-band filters in March 2014. The HSC-SSP will use 300 nights of Subaru time over about six years. The survey consists of three layers of different solid angles, going to different depths. With both the broad- and narrow-band photometric data, we will explore galaxy evolution over the full range of cosmic history from the present to redshift 7 . The measurement of galaxy shapes in the broad-band images will map the largescale distribution and evolution of dark matter through weak gravitational lensing (WL), and allow us to relate it to galaxy properties and distribution. Cross-correlations of HSC WL observables with the spectroscopic galaxy distribution in the Sloan Digital Sky Survey (SDSS; York et al. 2000)/Baryon Oscillation Spectroscopic Survey (BOSS; Dawson et al. 2013) and the observed temperature and polarization fluctuations in the Cosmic Microwave Background (CMB) will constrain the parameters of the standard model of cosmology, and test for exotic variations such as deviations from the predictions of General Relativity on cosmological scales (see Weinberg et al. 2013 for a review). Studies of the highest-redshift galaxies and quasars discovered in this survey will lead to a deeper understanding of reionization, a key event in the thermal history of the Universe.

The HSC survey follows a long tradition of major imaging surveys in astronomy. In the modern era, the Sloan Digital Sky Survey (SDSS; York et al. 2000) imaged one third of the celes- 
tial sphere with CCDs in five broad bands (ugriz), going to a depth of $r \approx 22.5$. The next generation of imaging surveys has surpassed SDSS in various combinations of depth, solid angle coverage, and image quality. For example, the Pan-STARRS1 survey (Chambers et al. 2016) used a 1.8-meter telescope to cover three-quarters of the sky in grizy almost a magnitude fainter than SDSS. DECaLS (Blum et al. 2016) is covering $14,000 \mathrm{deg}^{2}$ in $g r z$, going somewhat deeper than Pan-STARRS, and is designed to support the Dark Energy Spectroscopic Instrument (DESI, DESI Collaboration et al. 2016). The Dark Energy Survey (Dark Energy Survey Collaboration et al. 2016) is imaging 5,000 $\mathrm{deg}^{2}$ of the southern sky in five bands with the Blanco 4-meter telescope, going to $r \approx 24.3(10 \sigma)$. Weak lensing cosmology is a key driver of DES, and similarly is a driver of many of the more recent surveys. For example, the CFHT Lens Survey (Heymans et al. 2012) covers $154 \mathrm{deg}^{2}$ in five bands, to $i^{\prime}=25.5$. The Kilo-Degree Survey (KiDS; de Jong et al. 2017) is covering $1,500 \mathrm{deg}^{2}$ in 4 bands to $r=24.9$. The HSC survey described in this paper goes deeper than all these surveys, while still covering well over 1,000 $\mathrm{deg}^{2}$, and including a narrow-band imaging component as well.

This is the first paper in a series describing the HSC survey and its science in a special issue of the Publications of the Astronomical Society of Japan. Other key papers in this issue include a technical description of the HSC instrument itself (Miyazaki et al. 2017) and the software pipelines that analyze the data (Bosch et al. 2017; Huang et al. 2017, Murata et al. 2017). The first year of the data covering over $100 \mathrm{deg}^{2}$ in five broad bands have been released to the public, including fully reduced and calibrated images as well as catalogs of detected objects. The data release is described in Aihara et al. (2017) (hereafter the HSC DR1 paper). A separate analysis and catalog of galaxy shapes, crucial for weak lensing analysis, is included in Mandelbaum et al. (2017). This special issue also includes more than two dozen science papers based on the early data from the HSC survey, on topics ranging from asteroids to dwarf companions of the Milky Way, to weak lensing measurements of clusters, to some of the highest redshift quasars known.

We summarize the characteristics of the HSC instrument itself in $\S 2$. The survey design is described in $\S 3$, and the observing strategy follows in $\S 3.2$ and $\S 3.3$. $\S 4$ gives a brief overview of the data processing. We summarize, with a view to the future, in $\S 5$.

\section{Hyper Suprime-Cam}

While there are other 8-meter class telescopes around the world, Subaru is the one with by far the largest field of view. SuprimeCam (Miyazaki et al. 2002), with its $\sim 0.25 \mathrm{deg}^{2}$ field of view and superb delivered image quality (routinely $0.6^{\prime \prime}$ FWHM), has been a world leader in wide-field studies of the distant

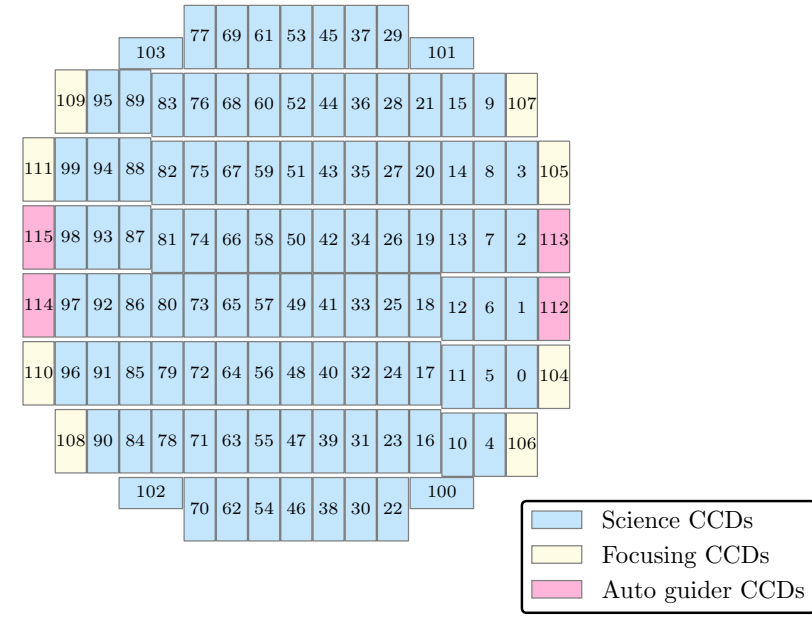

Fig. 1. The layout of 116 CCD chips, each of which has $2048 \times 4096$ pixels $\left(5.73^{\prime} \times 11.47^{\prime}\right)$, on the focal plane. The CCD chips are arranged with two different gaps of approximately $12^{\prime \prime}$ and $53^{\prime \prime}$ between the neighboring chips. The focal plane is approximately $1.5^{\circ}$ in diameter. There are 104 science chips (indicated in blue), 4 chips used for auto-guiding (in yellow) and 8 chips for monitoring the focus (in light red). Each chip is identified with a number from 0 to 115 .

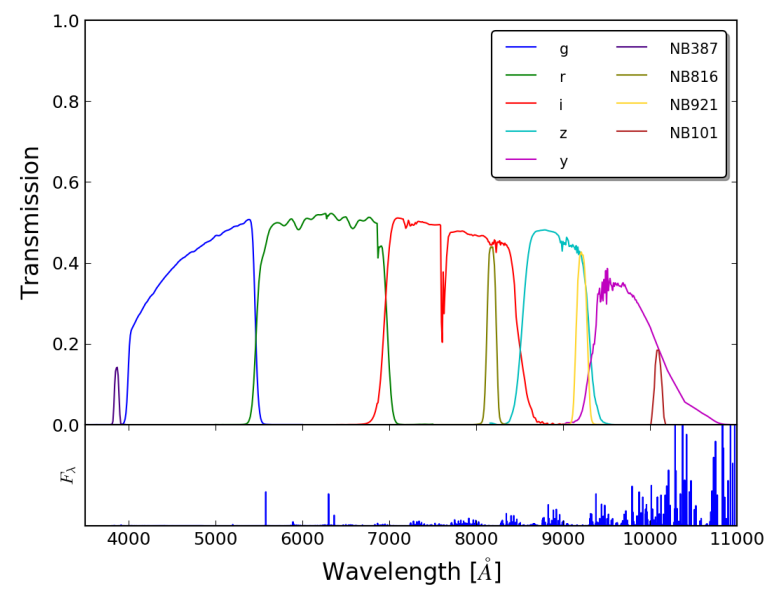

Fig. 2. The HSC bandpasses, including the reflectivity of all mirrors, transmission of all optics and filters as well as the atmosphere, and response of the CCDs, assuming an airmass of 1.2. Both the broad-band and narrow-band filters used in the survey are shown. The lower panel shows the spectrum of sky emission lines, demonstrating that the red narrow-band filters lie in relatively dark regions of the sky spectrum. 
and faint Universe (e.g., Iye et al. 2006; Furusawa et al. 2008). Hyper Suprime-Cam (HSC), its successor, takes advantage of the full accessible field of view of the Subaru telescope $\left(1.5^{\circ}\right.$ diameter), and thus has a survey power about 8 times larger than that of Suprime-Cam. The speed with which a given facility can survey the sky to a given depth is proportional to the product of the collecting area of the telescope and the field-ofview of the camera (étendue), although it also depends on image quality and the fraction of time any given facility is devoted to survey work. The étendue of HSC is the largest of all existing wide-field optical imaging cameras, not to be surpassed until the Large Synoptic Survey Telescope (LSST; LSST Science Collaboration et al. 2009; Ivezic et al. 2008) sees first light in late 2019.

The HSC instrument (Miyazaki et al. 2015, Miyazaki et al. 2017) was designed and built by an international team involving scientists from NAOJ, the Kavli Institute for the Physics and Mathematics of the Universe (Kavli IPMU), the University of Tokyo, the Academic Sinica Institute of Astronomy and Astrophysics in Taiwan (ASIAA), and Princeton University. The instrument has a large and optically very sophisticated seven-element Wide-Field Corrector (WFC), designed and built by Canon, Inc. The WFC incorporates an Atmospheric Dispersion Corrector (ADC) and delivers an instrumental PointSpread Function (PSF) for which the diameter enclosing $80 \%$ of the light $\left(D_{80}\right)$ is $0.2^{\prime \prime}$ or better over the entire field in all filters. A Prime Focus Unit (PFU) built by Mitsubishi Electric Corporation, which incorporates a precise hexapod for attitude and focus adjustment, holds the WFC and the camera in place at the telescope prime focus. The entire structure is roughly 3 meters tall, and weighs almost 3 tons. The corrector gives an unvignetted field of view to a diameter of 10 arcmin; vignetting is a roughly linear function of field radius from that point, reaching $26 \%$ at the edge of the field at $0.75^{\circ}$. The Subaru top-end structure has been modified to accommodate the PFU and WFC. The WFC can be used by other wide-field instruments as well, and is incorporated into the design of the planned Prime Focus Spectrograph (Takada et al. 2014; Tamura et al. 2016).

Table 1 summarizes the characteristics of the HSC instrument. The focal plane is paved with a total of 116 Hamamatsu Deep Depletion CCDs, each $2 \mathrm{~K} \times$ $4 \mathrm{~K}$ pixels. The layout of the $\mathrm{CCD}$ chips is shown in Figure 1 (see https://www.subarutelescope.org/ Observing/Instruments/HSC/ccd.html for details). The $15 \mu \mathrm{m}$ pixels subtend about $0.168^{\prime \prime}$ on the sky, with some modest variation over the focal plane. Four of the CCDs are used for guiding and eight for automatically monitoring focus, leaving 104 science detectors. These chips are three-side buttable and each have four independent readout amplifiers. Gaps between chips are small, typically $12^{\prime \prime}$ in one dimension and $53^{\prime \prime}$ in the other. Regions of the focal plane more than 49 arcmin from the center are masked, giving an effective area of about $1.77 \mathrm{deg}^{2}$. The chips have excellent characteristics: low read noise, excellent charge transfer efficiency, few cosmetic defects, and most importantly, high quantum efficiency ( $>40 \%$ ) from $4000 \AA$ to $10,000 \AA$ (blueward of $4000 \AA$, the response is limited both by the CCDs and the optical elements in the WFC). The model system response, including reflectivity and transmission of all optics, is shown in Figure 2. Kawanomoto et al. (2017) give a detailed description of the filters used in the HSC SSP. With $0.168^{\prime \prime}$ pixels, the images are well-sampled in even the best seeing seen on the instrument, $0.4^{\prime \prime}$.

Table 3 lists the characteristics of the filters used in the HSC-SSP survey. The effective wavelength is defined following Schneider et al. (1983) as

$$
\lambda_{\mathrm{eff}}=\exp \left[\frac{\int d \ln \lambda S(\lambda) \ln \lambda}{\int d \ln \lambda S(\lambda)}\right],
$$

where $S(\lambda)$ is the throughput of the telescope, camera, filter and atmosphere as a function of wavelength (as plotted in Figure 2). We characterize the width of the filters in two ways. The full-width at half-maximum (FWHM) is a standard measure. Alternatively, we can follow Schneider et al. (1983) to define the dimensionless quantity:

$$
\sigma^{2}=\frac{\int d \ln \lambda S(\lambda) \ln ^{2}\left(\lambda / \lambda_{\mathrm{eff}}\right)}{\int d \ln \lambda S(\lambda)} .
$$

In the limit of a narrow top-hat filter:

$$
\mathrm{FWHM}=\lambda_{\text {eff }}\left(e^{\sqrt{3} \sigma}-e^{-\sqrt{3} \sigma}\right) .
$$

We also tabulate the effective filter throughput $Q$, defined as the integral of $S(\lambda)$ over $\ln \lambda$ (i.e., the denominator of equations 1 and 2).

The instrument is installed at prime focus using the existing Top Unit Exchanger (TUE) instrument handler, though modifications were necessary to ensure that the instrument is not damaged during installation.

The camera has a roll-type shutter, with excellent timing accuracy, allowing uniform exposure time over the field of view. Including readout and all overheads, the minimum time between exposures is about 35 seconds, allowing for efficient surveying of the sky. The overhead for telescope slewing is negligible, because we can move the telescope to the next target field during the camera readout. The filter exchange mechanism can hold six filters at one time. With the telescope at zenith, changing filters takes about 10 minutes. However, for safety reasons the primary mirror cover needs to be closed and instrument rotated to a fiducial angle before the filters can be changed, meaning that it takes about 30 minutes in practice between the end of one sky exposure and the start of the following exposure in a different filter.

The HSC survey uses five broad-band filters (grizy) modeled on the SDSS filter set (Figure 2 and Table 3), as well as four narrow-band filters sensitive to emission lines such as the 
Table 1. Hyper Suprime-Cam Characteristics

\begin{tabular}{|c|c|}
\hline Instrument weight & $\sim 3.2$ tons \\
\hline Field of View & $1.5^{\circ}$ diameter \\
\hline Vignetting & 0 at $0.15^{\circ} ; 26 \%$ at edge \\
\hline Pixel scale & $15 \mu \mathrm{m} \simeq 0.168^{\prime \prime}$ \\
\hline Detectors & Hamamatsu Photonics KK 2048x4096 (S10892-02) \\
\hline Number of CCDs & 104 science, 4 autoguide, 8 for monitoring the focus ${ }^{(1)}$ \\
\hline CCD QE & $40 \%$ at $4000 \AA, 10,000 \AA ; 95 \%$ at peak (at $-100^{\circ} \mathrm{C}$ ) \\
\hline CTE & 0.999999 \\
\hline Gain & $3.0 e^{-1} / \mathrm{ADU}$ \\
\hline Readnoise & $4.5 \mathrm{e}^{-}$ \\
\hline Readout & $20 \sec ^{(2)}$ \\
\hline Saturation level & $150,000 \mathrm{e}^{-}$ \\
\hline Filters & grizy +4 narrow-band ${ }^{(3)}$ \\
\hline Filter Exchanger & 6 filters installed at a time \\
\hline Filter Exchange Time & $\sim 30$ minutes $^{(4)}$ \\
\hline
\end{tabular}

NOTES - (1) The camera has 104 science CCDs, 4 CCDs for auto-guiding, and 8 CCDs for monitoring the focus (also see http: //subarutelescope . org/Observing/Instruments/HSC/parameters.html). (2) $20 \mathrm{sec}$ is the time needed for reading out signals from CCDs. The actual overhead time, the time from the end of the previous exposure to the beginning of the next exposure, is about $35 \mathrm{sec}$, which includes time needed for transferring data from the instrument to the data-taking computers, slewing the telescope between dithering positions, and so on. (3) The survey described in this paper uses five broad-band and four narrow-band filters, but there are additional filters available for use with HSC. (4) Before exchanging filters in the filter exchange unit, one must first move the telescope to zenith, rotate the instrument to a fiducial angle, and close the primary mirror cover.

\begin{tabular}{l|lllll}
\hline \hline Layer & $\begin{array}{l}\text { Area } \\
{\left[\mathrm{deg}^{2}\right]}\end{array}$ & $\begin{array}{l}\text { \# of } \\
\text { pointings }\end{array}$ & Filters \& Depth & $\begin{array}{l}\text { Volume } \\
{\left[h^{-3} \mathrm{Gpc}^{3}\right]}\end{array}$ & Key Science \\
\hline Wide & 1400 & 916 & grizy $(i \simeq 26)$ & $\sim 4.4(z<1.5)$ & WL Cosmology, $z \sim 1$ gals, Clusters \\
Deep & 26 & 15 & grizy+3NBs $(i \simeq 27)$ & $\sim 0.5(1<z<5)$ & $z \lesssim 2$ gals, SNeIa, WL calib. \\
UltraDeep & 3.5 & 2 & grizy+3NBs $(i \simeq 28)$ & $\sim 0.07(2<z<7)$ & high- $z$ gals (LAEs, LBGs), SNeIa \\
\hline
\end{tabular}

Table 2. Summary of HSC-Wide, Deep and UltraDeep layers.

\begin{tabular}{lllll}
\hline \hline Filter & $\lambda_{\text {eff }}(\AA)$ & $\sigma$ & FWHM $(\AA)$ & $Q$ \\
\hline$g$ & 4754 & 0.0873 & 1395 & 0.1590 \\
$r 2$ & 6175 & 0.0711 & 1503 & 0.1667 \\
$i 2$ & 7711 & 0.0599 & 1574 & 0.1149 \\
$z$ & 8898 & 0.0263 & 766 & 0.0487 \\
$y$ & 9762 & 0.0316 & 783 & 0.0352 \\
$r$ (old) & 6178 & 0.0694 & 1495 & 0.1505 \\
$i$ (old) & 7659 & 0.0597 & 1523 & 0.1154 \\
NB387 & 3863 & 0.0049 & 55 & 0.0026 \\
NB816 & 8177 & 0.0049 & 113 & 0.0074 \\
NB921 & 9214 & 0.0050 & 134 & 0.0075 \\
NB101 & 10092 & 0.0039 & 91 & 0.0018 \\
\hline \hline
\end{tabular}

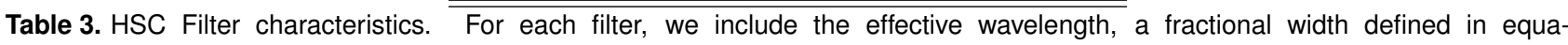
tion (2), the FWHM, and a measure of total throughput, as defined in the text. Note that we include the characteristics both of the updated $r$ and $i$ filters $(r 2$ and $i 2)$ as well as the old versions used in the early part of the survey. 
Lyman- $\alpha$ line over a wide range of redshifts. Given the filter exchange time, we usually do no more than two filter exchanges per night in survey mode.

HSC saw astronomical first light in 2012 August, and became a general user instrument for Subaru in March 2014, when the survey described here began. The original $r$ and $i$-band filters installed in the camera did not meet our full specifications, and we replaced them with filters with significantly more uniform response across the focal plane. We have used the new $i$-band filter (HSC- $i 2$ ) since Feb 2016 and the new $r$-band filter (HSC-r2) since Jul 2016 ${ }^{1}$. The DR1 data were all taken with the older versions of the filters.

\section{Survey design}

The HSC-SSP has been awarded 300 nights. We originally planned to observe 60 nights a year for five years starting in 2014, but the first two years of observing were allocated substantially less than this amount, as the instrument was operated under a shared-risk mode, available only for a few months each semester, while the Subaru Observatory was refining its techniques for mounting, operating, and unmounting this complex instrument. Thus the survey is likely to continue into 2019.

As described above, the principal scientific goals of the survey are studies of the distribution of dark matter in the universe from WL measurements, and probing the evolution of galaxies. This motivates surveying in three different modes, or layers: Wide, Deep, and UltraDeep, as summarized in Table 2.

While the Wide, Deep and UltraDeep layers will all take data in the same five broad-band filters, grizy (Figure 2), they use different narrow-band filters, they go to different depths, and they cover different solid angles and thus cosmological volumes. Table 4 summarizes the survey parameters of each layer. The survey depths are defined as the $5 \sigma$ PSF magnitude limit for isolated point sources, using photometric errors determined by the imaging pipeline (Bosch et al. 2017; see also the discussion in the HSC DR1 paper). The saturation limits depend on the individual visit exposure times, the seeing, and the sky brightness and transparency. For point sources in typical conditions for the exposure times in the Wide layer, the saturation limits in grizy are roughly $17.8,17.8,18.4,17.4$, and 17.1 , respectively. These are for median seeing of $0.72^{\prime \prime}, 0.67^{\prime \prime}, 0.56^{\prime \prime}, 0.63^{\prime \prime}$ and $0.64^{\prime \prime}$, respectively; the saturation limit in seeing a factor $f$ larger will be fainter by $5 \log f$ magnitudes. Similarly, the saturation limit in the 30-second exposures (Section 3.3) is brighter by $2.5 \log g$ magnitudes, where $g$ is the ratio of the single-visit exposure time to 30 seconds (corresponding to 1.75 mags in $g r$ and 2.06 mags in $i z y$ in the wide survey). Finally, the throughput of the system is proportional to the quantity $Q$ tabulated in

\footnotetext{
${ }^{1}$ http://subarutelescope.org/Observing/Instruments/HSC/ sensitivity.html
}

Table 3, which allows one to calculate approximate saturation limits in the narrow-band filters as well.

\subsection{Survey fields}

Table 5 summarizes the target fields for the HSC-Wide, Deep and UltraDeep layers (also see Figure 3). The fields are chosen to overlap the footprint of the Sloan Digital Sky Survey (York et al. 2000; Aihara et al. 2011) and the Pan-STARRS1 survey (Chambers et al. 2016), as we use them for the first-order photometric and astrometric calibration. In the following we describe details of the target fields and the rationale for selecting these fields for each layer.

\subsubsection{HSC Wide layer}

One of the primary science drivers for the HSC-Wide layer is to explore the nature of dark matter and dark energy via WL observables (e.g., Weinberg et al. 2013; Takada \& Jain 2004; Oguri \& Takada 2011). We will primarily use the $i$-band data to perform the galaxy shape measurements. We perform $i$-band observations in the Wide layer when the weather is clear and the seeing is good, in order to perform accurate shape measurements of galaxies (see below for details). At a depth of $i \approx 26$, we predict a weighted mean number density of galaxies for which shapes can be measured of $\bar{n}_{\text {eff }} \simeq 20 \operatorname{arcmin}^{-2}$, with a mean redshift of $\langle z\rangle \simeq 1$. Combining the $i$-band data with the gr zy photometry will allow us to estimate photometric redshifts (photo- $z$ ) for every galaxy used in the WL analysis; the relative depths of the different bands are selected to optimize the photo$z$ accuracy (Tanaka et al. 2017). A solid angle of 1,400 $\mathrm{deg}^{2}$ will give us the statistical precision for the WL observables to obtain a tight constraint on dark energy parameters at a similar level of precision to that of Stage-III dark energy experiments (Mandelbaum et al. 2017).

The Wide layer sky coverage is mostly along the Celestial Equator (making the fields easily observable from both hemispheres). The Wide layer fields stretch over a wide range of right ascension, such that fields are reachable at all times of the year. We selected regions of sky low in Galactic extinction, away from the disk of the Milky Way. The survey footprint includes three large spatially contiguous regions, to enable cosmological analyses on large scales. Figure 3 and Table 5 show the survey footprint, which consists of three parts, termed "the fall equatorial field", "the spring equatorial field" and "the north field". Our selection of these regions is aimed to overlap other multi-wavelength data to maximize the scientific synergy with HSC. In particular, we consider the arc-minute-resolution, highsensitivity CMB survey by the Atacama Cosmology Telescope (ACT; Swetz et al. 2011) in Chile, and its polarization extension ACTPol (Thornton et al. 2016); X-ray data from the 


\begin{tabular}{|c|c|c|c|c|c|c|c|c|c|}
\hline Wide & $g$ & $r$ & $i$ & $\bar{z}$ & $y$ & & & & \\
\hline total exp. (min) & 10 & 10 & 20 & 20 & 20 & & & & \\
\hline exp. per visit $(\mathrm{min} / \mathrm{sec})$ & 2.5 & 2.5 & $200 \mathrm{sec}$ & $200 \mathrm{sec}$ & $200 \mathrm{sec}$ & & & & \\
\hline depth (mag) & 26.5 & 26.1 & 25.9 & 25.1 & 24.4 & & & & \\
\hline Deep & $g$ & $r$ & $i$ & $z$ & $y$ & NB387 & NB816 & NB921 & \\
\hline exp. (min) & 84 & 84 & 126 & 210 & 126 & 84 & 168 & 252 & \\
\hline exp. per visit (min) & 3 & 3 & 4.5 & 4.5 & 4.5 & 14 & 15 & 15 & \\
\hline depth (mag) & 27.5 & 27.1 & 26.8 & 26.3 & 25.3 & 24.5 & 25.8 & 25.6 & \\
\hline 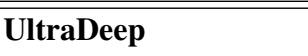 & $g$ & $\overline{c r}$ & $\overline{\bar{i}}$ & $\bar{z} z$ & $\bar{y}$ & & NB816 & NB921 & $\overline{~ N B 101}$ \\
\hline $\exp .(\min )$ & 420 & 420 & 840 & 1134 & 1134 & - & 630 & 840 & 1050 \\
\hline exp. per visit (min) & 5 & 5 & 5 & 5 & 5 & - & 15 & 15 & 15 \\
\hline depth (mag) & 28.1 & 27.7 & 27.4 & 26.8 & 26.3 & - & 26.5 & 26.2 & 24.8 \\
\hline
\end{tabular}

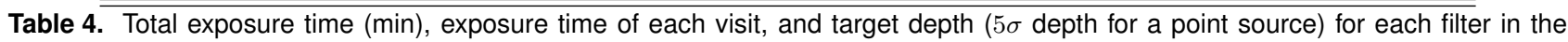
Wide, Deep and UltraDeep layers, respectively. Dividing the total exposure time by each visit exposure time gives the number of visits for each field. For example, in the Wide layer, there are four visits for each field in $g$ and $r$, and six visits in $i, z$, and $y$.

\begin{tabular}{|c|c|c|}
\hline Layer & Field name & (RA, Dec) \\
\hline \multirow[t]{3}{*}{ Wide } & North field & $13: 20 \leq \mathrm{RA} \leq 16: 40 \& 42^{\circ} \leq \operatorname{dec} \leq 44.5^{\circ}\left(\simeq 90 \mathrm{deg}^{2}\right)$ \\
\hline & Spring equatorial field & $08: 30 \leq \mathrm{RA} \leq 15: 00 \&-2^{\circ} \leq \mathrm{dec} \leq+5^{\circ}\left(\simeq 680 \mathrm{deg}^{2}\right)$ \\
\hline & Fall equatorial field & $22: 00 \leq \mathrm{RA} \leq 02: 40 \&-1^{\circ} \leq \operatorname{dec} \leq 7^{\circ}\left(\simeq 630 \mathrm{deg}^{2}\right)$ \\
\hline \multirow[t]{15}{*}{ Deep } & XMM-LSS & $(02: 23: 15.33,-05: 18: 30.67)$ \\
\hline & & $(02: 22: 22.43,-04: 03: 44.48)$ \\
\hline & & $(02: 27: 19.86,-04: 27: 47.42)$ \\
\hline & E-COSMOS & $(09: 57: 28.60,+02: 57: 21.00)$ \\
\hline & & $(10: 03: 28.60,+02: 57: 21.00)$ \\
\hline & & $(09: 57: 28.60,+01: 27: 21.00)$ \\
\hline & & $(10: 03: 28.60,+01: 27: 21.00)$ \\
\hline & ELAIS-N1 & $(16: 11: 00.81,+53: 56: 30.71)$ \\
\hline & & $(16: 15: 46.66,+54: 59: 17.74)$ \\
\hline & & $(16: 10: 54.07,+56: 02: 49.65)$ \\
\hline & & $(16: 06: 16.19,+54: 59: 16.91)$ \\
\hline & Deep2-3 & $(23: 32: 08.46,+00: 16: 49.43)$ \\
\hline & & $(23: 27: 02.16,+00: 16: 49.41)$ \\
\hline & & $(23: 29: 26.53,-00: 50: 38.94)$ \\
\hline & & $(23: 24: 12.60,-00: 49: 46.61)$ \\
\hline \multirow[t]{2}{*}{ UltraDeep } & SXDS & $(02: 18: 15.60,-04: 51: 00.00)$ \\
\hline & COSMOS & $(10: 00: 28.60,+02: 12: 21.00)$ \\
\hline- & AEGIS & $(14: 17: 00.0,+52: 30: 00.00)$ \\
\hline
\end{tabular}

Table 5. List of the target fields; see Figures 3 and 4. The coordinates for the Deep and UltraDeep layers and the AEGIS (All-wavelength Extended Groth Strip; Davis et al. 2007) field are the centers of each fiducial pointing. The range of coordinates for the Wide layer defines the approximate boundaries of each survey field, although the exact boundary of the full depth region depends on the details of the dither pattern, as described in $\S$ 3.3. The numbers in the round brackets for the Wide layer give the area of each field. AEGIS is a single pointing to Wide layer depth in the five broad bands, useful for calibrating photometric redshifts. 

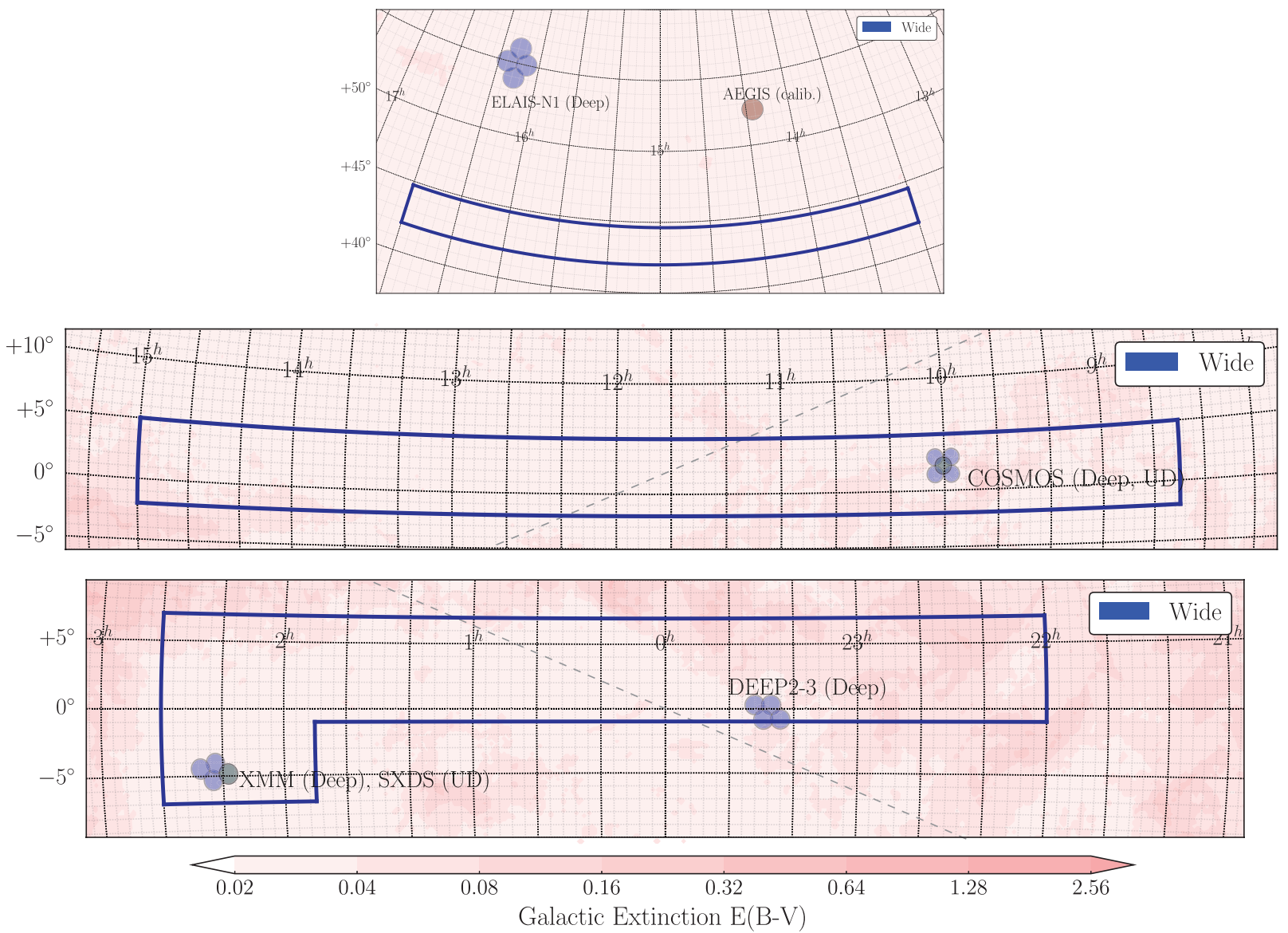

Fig. 3. The location of the HSC-Wide, Deep, and UltraDeep fields and the AEGIS field on the sky in equatorial coordinates. The color scale gives the level of Galactic dust extinction from Schlegel et al. (1998), as denoted by the color bar. See Figure 4 for the details of the Deep and UltraDeep fields.

XMM-XXL survey (Pierre et al. 2016) and eROSITA ${ }^{2}$; near/mid-infrared imaging surveys such as VIKING ${ }^{3}$ (Edge et al. 2013) and UKIDSS ${ }^{4}$ (Lawrence et al. 2007); and spectroscopic surveys such as SDSS-III/BOSS (Dawson et al. 2013), VVDS (Le Fèvre et al. 2013), VIPERS ${ }^{5}$ (Guzzo et al. 2014), GAMA ${ }^{6}$ (Driver et al. 2011), AEGIS (Davis et al. 2007), DEEP2 $/ 3^{7}$ ${ }^{8}$ (Newman et al. 2013), PRIMUS ${ }^{9}$ (Coil et al. 2011), and HectoMAP (Hwang et al. 2016).

\subsubsection{HSC Deep and UltraDeep layers}

The primary science goals of the HSC-Deep and UltraDeep layers are the study of galaxy and AGN evolution over cosmic time, and a survey for high-redshift supernovae as a cosmological probe. The UltraDeep regions are single pointings in the Deep fields, and (with one exception, see below) the Deep fields are included in the Wide layer fields (see Figure 3). The

\footnotetext{
${ }^{2}$ http://www.mpe.mpg.de/eROSITA

${ }^{3}$ http://www. astro-wise.org/projects/VIKING/

${ }^{4}$ http://www.ukidss.org

${ }^{5}$ http://vipers.inaf.it/papers.html

${ }^{6}$ http://www.gama-survey.org

${ }^{7}$ http://deep.ps.uci.edu/

${ }^{8}$ http://deep.ps.uci.edu/deep3/home.html

${ }^{9}$ http://cass.ucsd.edu/ acoil/primus/Home.html
}

Deep and UltraDeep components of the survey are driven by several requirements. The first is to study high-redshift galaxies, including Lyman-break galaxies selected by their broadband colors, and Lyman- $\alpha$ emitters with emission lines falling into the narrow-band filters corresponding to redshifts 2.2, 5.7, 6.6, and 7.3. The second is to explore the variable universe in the Deep and UltraDeep fields, especially searching for $z \gtrsim 1$ supernovae. At redshifts of 6 and above, the spatial and luminosity distribution of galaxies holds important clues about the timing and topology of cosmic reionization. The Deep and UltraDeep data will have significantly higher signal-to-noise ratio for galaxies at the limits of the Wide layer imaging, making them ideal for testing systematics in shape and photometric measurements (see Table 4).

Our four Deep layer fields are listed in Table 5 and are shown in Figure 4. DEEP2-3 and ELAIS-N1 each have four pointings, while XMM-LSS has three, overlapping with a fourth pointing to UltraDeep depths, SXDS. There are four E-COSMOS pointings to Deep depth, which overlaps with a fifth pointing to UltraDeep depth, $\operatorname{COSMOS}^{10}$. The four fields are all regions which already have extensive multi-wavelength imaging and

${ }^{10}$ http: //cosmos.astro.caltech.edu 

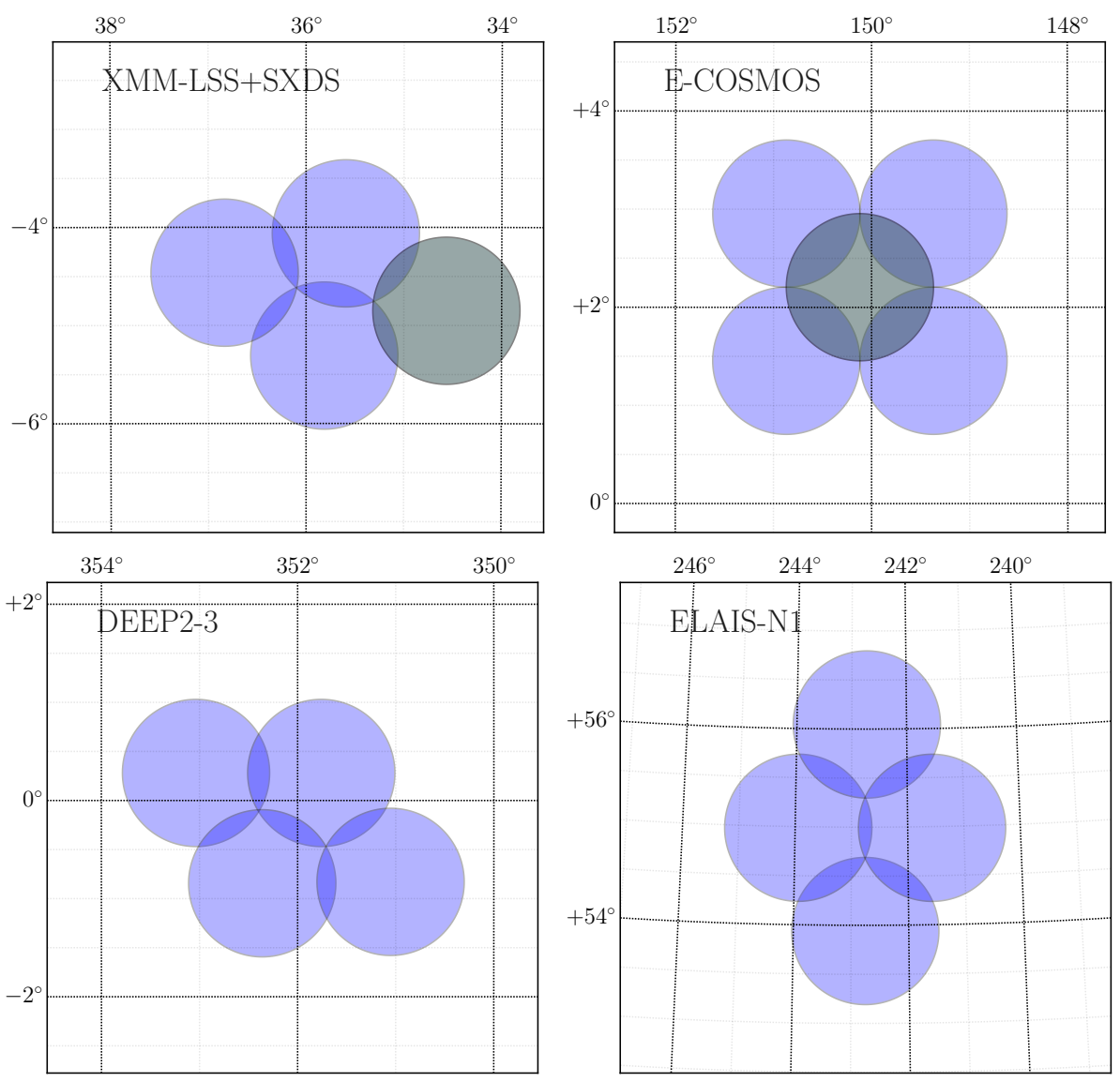

Fig. 4. The blue and dark-green circles show locations of the fiducial pointings of the Deep and UltraDeep fields, respectively (see Table 5). We have an additional five dithered pointings around each fiducial pointing, as described in the text.

spectroscopy. The ELAIS-N1 field does not lie in the Wide footprint, but it has deep NIR data from the UKIDSS-DXS (Lawrence et al. 2007), and is one of the deep LOFAR (van Haarlem et al. 2013) survey fields. LOFAR uses an array of omni-directional antennas designed to detect the $21 \mathrm{~cm}$ signals from neutral hydrogen in the cosmic reionization epoch. The cross-correlation of our HSC Lyman- $\alpha$ emitter sample with the LOFAR data will allow us to explore the relationship of reionization to the LAE distribution. We are gathering additional multi-band data in the Deep and UltraDeep areas of the sky, including $u$-band observations with the Canada-France-Hawaii Telescope (Wang et al. in prep., Sawicki et al. in prep.), and near-infrared data using the United Kingdom Infrared Telescope (UKIRT; Egami et al. in prep.). These data supplement existing VIDEO data from the VISTA telescope (Jarvis et al. 2013), as well as deep pointings in the UltraDeep fields with the Spitzer telescope (Steinhardt et al. 2014).

In the UltraDeep layer, we will carry out the deepest HSC imaging for a total area of $3.5 \mathrm{deg}^{2}$ in two independent blank fields well separated on the sky, each covered with one pointing of HSC, and each overlapping a Deep field. Targeting two fields will yield a large sample of high- $z$ supernovae and galaxies, and will allow us to evaluate cosmic variance in all statistical measurements we make in the two fields.

\subsection{Observing Strategy}

The HSC-SSP comprises an interlocking set of observations in five broad-band and four narrow-band filters, and with three different layers to different depths. Carrying out efficient observations through the full 300 nights of the survey requires careful planning, with an aim to making the data immediately useful to HSC scientists as the survey progresses.

The HSC instrument is mounted at the Prime Focus of the Subaru telescope for each run. Runs are typically two weeks long, centered on New Moon. The filter exchanger can hold six filters at a given time, and the complement of filters cannot be changed during a run. Moreover, because the filter exchanger is installed the day after the instrument is installed, and removed the day before the instrument is removed, only one filter is available for observations on the first and last day of any given run. The two-week runs include observations for the HSC-SSP, as well as a variety of general-use programs, so the choice of filters to be installed in the exchanger for any given run must balance 

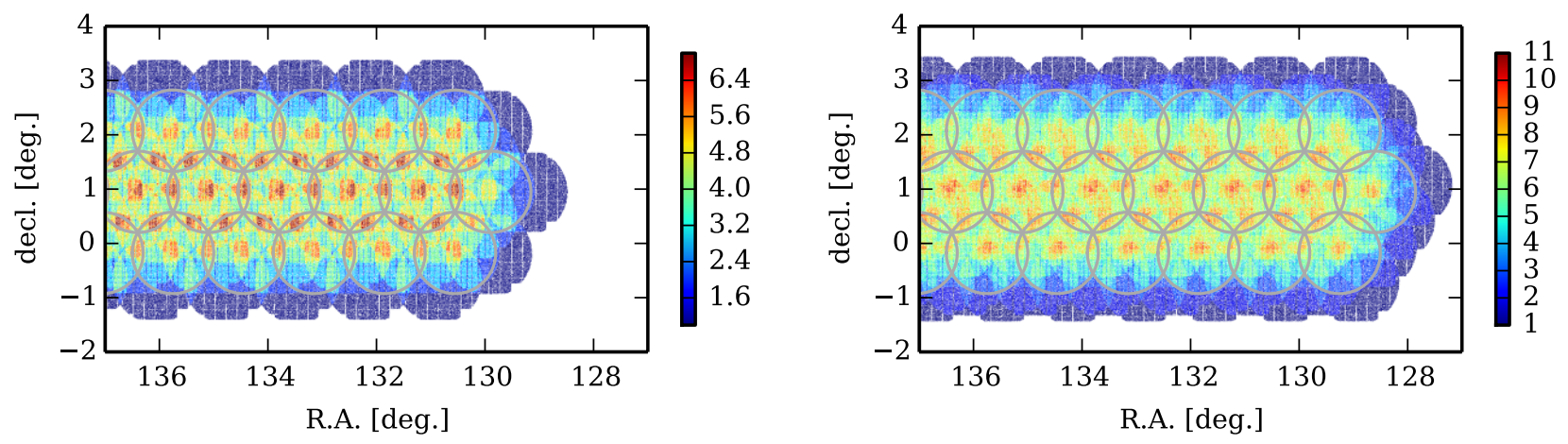

Fig. 5. The spatial distribution of the number of visits in an example region of the Wide layer. The left and right panels show the coverage in the $g$ and $z$ bands, respectively. The solid circles show the fiducial pointings around which dithering is carried out (4 and 6 visits for $g r$ or $i z y$, respectively). Full depth for the Wide layer corresponds to greenish regions in each panel.
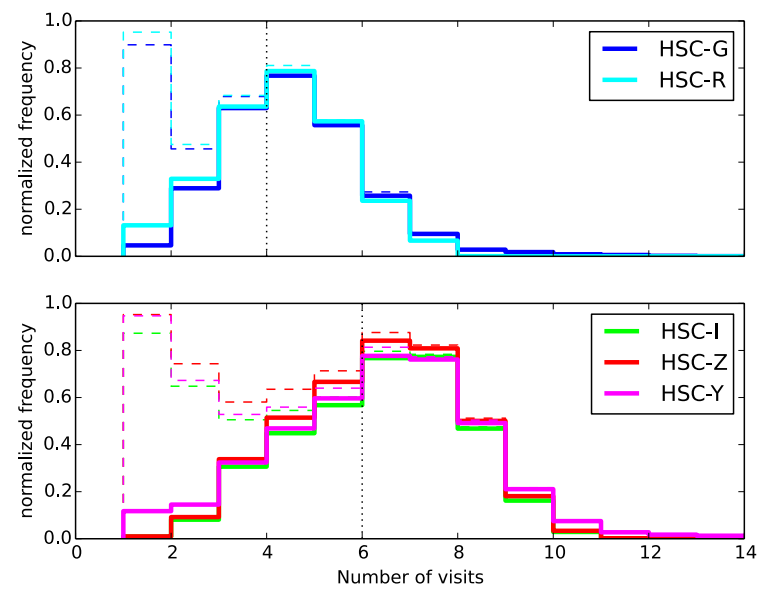

Fig. 6. Distribution of the number of exposures for different dithering patterns within the Wide layer footprint. $g$ and $r$-band are shown in the upper panel, and $i, z$ and $y$ in the lower panel, taken from the HSC DR1 data. The vertical dotted lines represent our target number of exposures; note that because of the dithering, a significant fraction of the area gets more exposures than this. Dashed histograms are for the entire region, while the solid are for regions inside the fiducial pointings only. The fiducial pointings are shown in Figure 5.

the needs of all these programs. On any given run, the filter exchanger typically holds four or all five of the broad-band filters, and one or two of the narrow-band filters.

As described in the HSC DR1 paper, in the early phases of the survey we have focused on regions of the sky with extensive external datasets, to calibrate and validate our data and to maximize scientific synergy. These included the GAMA, AEGIS, VVDS, VIPERS and DEEP2-3 regions, where extensive spectroscopic data are available, and the COSMOS field, where accurate 30-band photometric redshifts are also available. In the XMM-LSS region, we also have X-ray data from the XMMLSS survey (Pierre et al. 2016) and Sunyaev-Zel'dovich data from ACTPol (Niemack et al. 2010), which are complementary to optically selected (Oguri et al. 2017a) or weak-lensing selected clusters (Miyazaki et al. 2015; Oguri et al. 2017b) in
HSC. The UKIDSS and VIKING surveys have also carried out deep near-infrared imaging in the equatorial fields, which is particularly useful for quasar studies (Toba et al. 2015; Matsuoka et al. 2016).

In the first years of the HSC SSP survey, we have aimed to reach full depth in any given region of the Wide layer in all five filters fairly quickly (i.e., within a few lunations), and only then build up area with time. Having photometry in all five bands is crucial for most of the HSC-SSP survey goals. However, given the overhead in changing filters, we observe in no more than two filters most nights, occasionally using three filters when observing in the Deep and UltraDeep fields. As the survey matures, we are working to bridge already-observed fields in order to maximize the contiguous area in the survey footprint.

If a given night is dark, and clear weather and good seeing are forecasted, we usually start our observation in the $i$ band, in which we will do our WL shape measurements. We require that the $i$-band data be taken with seeing better than $0.8^{\prime \prime}$. If the seeing becomes worse than this, we change filters. The $i$-band data we have taken mostly satisfies this condition; the median seeing in our $i$-band data is about $0.6^{\prime \prime}$ (the HSC DR1 paper). We perform observations in the redder filters, $z, y$, NB816, NB921, and NB101 when the Moon is up. The seeing in other bands is only somewhat worse, and a significant fraction of the HSC data in all bands has seeing better than $0.8^{\prime \prime}$ (the HSC DR1 paper).

Our survey design includes about $2 / 3$ of the observing time in the Wide layer, with $1 / 3$ for the Deep and UltraDeep observations combined. The narrow-band imaging in the Deep and UltraDeep fields can be carried out only in those observing runs when the appropriate filters have been loaded into the filter exchanger. In the first year, we aimed to observe in the Deep and UltraDeep layers to roughly $1 / 5$ of the ultimate depth. Starting in late 2016, we adopted a specific cadence for the UltraDeep broad-band observations to maximize the sensitivity to and measurement of the lightcurves of $z \gtrsim 1$ supernovae. We are planning to obtain about $60 \%$ of the total exposures in 


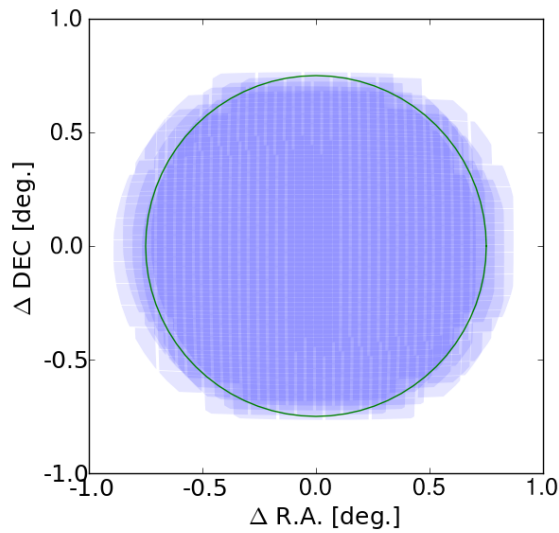

Fig. 7. The dithering pattern for one five-dithered pointing in each field of the Deep and UltraDeep layers. The circle has a radius of $0.75^{\circ}$. The intensity of the color is proportional to the number of visits. The figure includes the CCD gaps.

grizy in each of the UltraDeep COSMOS or SXDS fields during a single semester in 2017 or 2018 in a focused campaign to search for such supernovae. This will leave about $10 \%$ of the exposures to be carried out over the remainder of the survey.

Finally, for each run, we usually take basic calibration data (biases, darks and dome flats) for each of the filters installed in the filter exchange unit.

\subsection{Pointing Strategy}

We have quick-look analysis tools that allow us to determine the seeing and the sky transparency of each exposure while observing (Furusawa et al. 2017). The transparency is measured by comparing the observed brightness of stars in each field with those from the SDSS. This allows us to make decisions on the spot regarding the filter and area of sky to cover. We retake visits which do not satisfy our criteria of seeing $\left(<1.2^{\prime \prime}\right)$ or transparency $(>0.7)$ but the exposure time is always kept the same (see Table 4).

We also monitor the focus in close to real time using data from the focus chips, which are mounted slightly above and below the focal plane. When they show the instrument to be out of focus, we take a special set of short exposures over a range of focus positions, and adjust the focus accordingly.

In the Wide layer, the total exposure time is 10-20 minutes, depending on the filter (Table 2), divided up into 4-6 individual visits. We separate successive exposures in a given field by more than half an hour, in order to have independent realizations of the atmosphere and thereby average out atmospheric effects to some extent. To increase the dynamic range at the bright end, we also take a single 30-second exposure in each field and for each filter. This short exposure gives us many unsaturated stars in the magnitude range that SDSS and Pan-STARRS1 probe, crucial for the first-order astrometric and photometric calibra- tion.

Figure 5 demonstrates our pointing strategy to cover the target fields in the HSC-Wide layer. The gray circles are the "fiducial" pointings which define the survey geometry, each with a radius of $0.75^{\circ}$, approximately denoting the HSC field-of-view. We dither the telescope between exposures to homogenize the depth of the survey, fill gaps between CCD chips, improve measurement of scattered light, and control the photometric and astrometric calibration of the survey. We offset the telescope between successive exposures with a dither pattern parameterized by $\left(\Delta r_{\text {dith }}, \theta_{\text {dith }}\right)$, where $\Delta r_{\text {dith }}$ is the angular separation between the centers of the fiducial pointing and the dithered pointings, and $\theta_{\text {dith }}$ is the position angle from the west-east direction on the sky. We adopt $\Delta r_{\text {dith }}=0.6^{\circ}$ for the equatorial regions and $0.3^{\circ}$ for the northern sky field, which is smaller due to the narrower width of the field. We take $\theta_{\text {dith }}=\theta_{0}+\left(2 \pi / N_{\text {dith }}\right) \times j$ for the position angle for the $j$-th visit exposure; $j=0,1,2$ or $j=0,1, \cdots, 4$ for the $g r$ or $i z y$ filters, respectively. In order to have a homogeneous depth over different fields within the Wide layer we employed $\theta_{0} \simeq 63^{\circ}$ for $g r$ and $\theta_{0} \simeq 27^{\circ}$ for $i z y$. The color scales in Figure 5 show the number of visits (exposures) at each position.

Figure 6 shows the distribution of the number of visits to a given region of sky for the HSC DR1 Wide layer data in different filters. The dashed lines are for the entire survey footprint, while the solid lines are limited to the region within the fiducial pointings. Note that there are regions that go beyond the targeted number of exposures (four exposures in $g r$, and six exposures in $i z y$ ). In the DR1 catalog we define the area over which we have full-color, full-depth data in the Wide layer to be the intersection of the footprint in the five bands with more than a nominal number of visits (see Section 3.9 in the DR1 paper for details).

Because the Deep and UltraDeep fields are quite small (relatively speaking), we cannot take as large a dither as we do in the Wide layer. We instead carry out a five-pointing dither pattern to fill CCD gaps: we take five dithered pointings centered at $(\Delta \mathrm{RA}, \Delta \mathrm{Dec})=(0,0),(150,-150),(300,75),(-150,150)$, and $(-300,-75)(\operatorname{arcsec})$, around the fiducial pointing given in Table 5. In addition, for each set of five dithered pointings, we randomly offset the fiducial pointing within a 7.5 arcmin radius, roughly corresponding to a size of a CCD chip, to increase the uniformity in the field. In the Deep layer, the individual visit exposure times in $g$ and $r$ are 180 seconds, and 270 seconds in $i, z$ and $y$. We typically take 3-5 exposures for each field in a given filter on a given night (as long as the weather allows). In the UltraDeep layer, the exposure time for each visit is 300 seconds for all bands, and we carry out 3-10 visits on a given night. We will continue these exposures through the lifetime of the survey until we reach the specified total exposure times (Table 4).

Figure 7 shows the dithering pattern for a single set of five 
exposures in the Deep or UltraDeep layer; the random shift is not included. The intensity of the color represents the number of exposures covering that pointing.

\section{Data processing and HSC pipeline}

The HSC-SSP data are processed through a software package, hscPipe, which is closely allied to the development of the software pipeline for the LSST (Axelrod et al. 2010; Jurić et al. 2015). The spirit of the HSC image processing is that most of the core science goals of the SSP can be carried out by the pipeline outputs. That is, the aim is that one will be able to do science investigations directly from the measured quantities of the pipeline, without needing to re-analyze the images themselves. The hscPipe pipeline is summarized in the HSC DR1 paper, and is described in detail in Bosch et al. (2017). As described in the HSC DR1 paper, the photometric calibration goal is $1 \%$ rms precision. External comparison with SDSS and PanSTARRS photometry shows that we are nearing that goal, but more work needs to be done. The astrometric calibration is good to 20 mas rms, although there are still systematic residuals that we are working to control.

For processing purposes, the sky is divided up on a predefined grid called tracts, each covering $1.7 \times 1.7$ square degrees of sky, and each tract is subdivided into $9 \times 9$ subareas, patches, squares roughly 12 arcminutes on a side. Each exposure of the CCD array is termed a visit. The data from each visit are corrected for cosmetic features, including bad pixels and columns, and are bias subtracted, flat-fielded, and corrected for chip non-linearity and the brighter-fatter effect, whereby the PSF of bright stars is larger due to electron spreading in the CCDs (Antilogus et al. 2014). Then the pipeline performs multi-visit processing to generate coadd images of multiple exposures. Objects are detected on the coadd images in each band separately, and the union of these objects are used to measure the photometric and astrometric properties across the bands.

The pipeline results depend critically on an accurate determination of the point spread function (PSF). This is important for the photometry of stars, for model fits to galaxies, for determination of galaxy shapes for weak lensing, and for modeling overlapping images (the deblending problem). We determine the PSF using a customized version of PSFex (Bertin 2011). The measured properties of all detected objects are stored in a postgreSQL database as described in Takata et al. (2017), while the image files are available for direct download. Please see Bosch et al. (2017) and the HSC DR1 paper for more details about the pipeline, including a description of known problems with the data and its processing. The details of the galaxy shape catalog used for the WL measurements are given in Mandelbaum et al. (2017).

In addition to hscPipe, we also use the HSC synthetic galaxy pipeline SynPipe (Huang et al. 2017; Murata et al. 2017). This is a Python-based module that interfaces with hscPipe and can inject realistic synthetic stars and galaxies at desired locations of single-visit HSC images. We use SynPipe to examine the photometric performance of hscPipe (Huang et al. 2017) as well as to characterize the effects of galaxy blends for the Wide survey (Murata et al. 2017).

\section{Conclusion}

This paper describes the design of a 300-night imaging survey of the sky with Hyper Suprime-Cam, a $1.77 \mathrm{deg}^{2}$ imaging camera mounted on the Prime Focus of the $8.2 \mathrm{~m}$ Subaru telescope. The survey will extend from 2014 through 2019, and is being done in five broad bands ( grizy) and four narrow bands. The survey has three layers, termed Wide, Deep, and UltraDeep, covering $1400 \mathrm{deg}^{2}, 26 \mathrm{deg}^{2}$, and $3.5 \mathrm{deg}^{2}$, respectively. As described in detail in the HSC DR1 paper, the survey data to date are of very high quality, with median seeing of $0.6^{\prime \prime}$ in the $i$-band, and only somewhat worse in the other bands. This paper introduces a special issue of the PASJ, with a combination of technical papers describing the instrument and survey, and science papers describing a broad range of exciting results from the first year of the survey. We anticipate future data releases in 2019 and in 2021.

The HSC-SSP survey is part of a larger project, termed "Subaru Measurements of Images and Redshifts" (SuMIRe). The HSC team, together with additional partners in the US, France, Germany, Brazil and China, are building a wide-field multi-object spectrograph (Takada et al. 2014; Tamura et al. 2016), which will use the same WFC as HSC. With it, we plan to carry out wide-field spectroscopic surveys of stars, galaxies, and quasars selected from the superb imaging data from the HSC-SSP survey.

\section{Acknowledgements}

The Hyper Suprime-Cam (HSC) collaboration includes the astronomical communities of Japan and Taiwan, and Princeton University. The HSC instrumentation and software were developed by the National Astronomical Observatory of Japan (NAOJ), the Kavli Institute for the Physics and Mathematics of the Universe (Kavli IPMU), the University of Tokyo, the High Energy Accelerator Research Organization (KEK), the Academia Sinica Institute for Astronomy and Astrophysics in Taiwan (ASIAA), and Princeton University. Funding was contributed by the FIRST program from Japanese Cabinet Office, the Ministry of Education, Culture, Sports, Science and Technology (MEXT), the Japan Society for the Promotion of Science (JSPS), Japan Science and Technology Agency (JST), the Toray Science Foundation, NAOJ, Kavli IPMU, KEK, ASIAA, and Princeton University. HM is supported by the 
Jet Propulsion Laboratory, California Institute of Technology, under a contract with the National Aeronautics and Space Administration. This paper makes use of software developed for the Large Synoptic Survey Telescope. We thank the LSST Project for making their code available as free software at http://dm.lsst.org.

The Pan-STARRS1 Surveys (PS1) have been made possible through contributions of the Institute for Astronomy, the University of Hawaii, the Pan-STARRS Project Office, the Max-Planck Society and its participating institutes, the Max Planck Institute for Astronomy, Heidelberg and the Max Planck Institute for Extraterrestrial Physics, Garching, The Johns Hopkins University, Durham University, the University of Edinburgh, Queen's University Belfast, the HarvardSmithsonian Center for Astrophysics, the Las Cumbres Observatory Global Telescope Network Incorporated, the National Central University of Taiwan, the Space Telescope Science Institute, the National Aeronautics and Space Administration under Grant No. NNX08AR22G issued through the Planetary Science Division of the NASA Science Mission Directorate, the National Science Foundation under Grant No. AST-1238877, the University of Maryland, and Eotvos Lorand University (ELTE) and the Los Alamos National Laboratory.

Based on data collected at the Subaru Telescope and retrieved from the HSC data archive system, which is operated by Subaru Telescope and Astronomy Data Center at National Astronomical Observatory of Japan.

This work is supported in part by JSPS KAKENHI (Grant Number JP 15H03654) as well as MEXT Grant-inAid for Scientific Research on Innovative Areas (15H05887, 15H05892, 15H05893, 15K21733).

\section{References}

Aihara, H., Allende Prieto, C., An, D., et al. 2011, ApJS, 193, 29

Aihara, H., Armstrong, R., Bickerton, S., et al. 2017, ArXiv e-prints, arXiv:1702.08449 (HSC DR1 paper)

Antilogus, P., Astier, P., Doherty, P., Guyonnet, A., \& Regnault, N. 2014, Journal of Instrumentation, 9, C03048

Axelrod, T., Kantor, J., Lupton, R. H., \& Pierfederici, F. 2010, in Proc. SPIE, Vol. 7740, Software and Cyberinfrastructure for Astronomy, 774015

Bertin, E. 2011, in Astronomical Society of the Pacific Conference Series, Vol. 442, Astronomical Data Analysis Software and Systems XX, ed. I. N. Evans, A. Accomazzi, D. J. Mink, \& A. H. Rots, 435

Blum, R. D., Burleigh, K., Dey, A., et al. 2016, in American Astronomical Society Meeting Abstracts, Vol. 228, American Astronomical Society Meeting Abstracts, 317.01

Bosch, J., Armstrong, R., Bickerton, S., et al. 2017, ArXiv eprints, arXiv: 1705.06766
Chambers, K. C., Magnier, E. A., Metcalfe, N., et al. 2016, ArXiv e-prints, arXiv: 1612.05560

Coil, A. L., Blanton, M. R., Burles, S. M., et al. 2011, ApJ, 741, 8

Dark Energy Survey Collaboration, Abbott, T., Abdalla, F. B., et al. 2016, MNRAS, 460, 1270

Davis, M., Guhathakurta, P., Konidaris, N. P., et al. 2007, ApJL, 660, L1

Dawson, K. S., Schlegel, D. J., Ahn, C. P., et al. 2013, AJ, 145, 10

de Jong, J. T. A., Verdoes Kleijn, G. A., Erben, T., et al. 2017, ArXiv e-prints, arXiv:1703.02991

DESI Collaboration, Aghamousa, A., Aguilar, J., et al. 2016, ArXiv e-prints, arXiv:1611.00036

Driver, S. P., Hill, D. T., Kelvin, L. S., et al. 2011, MNRAS, 413, 971

Edge, A., Sutherland, W., Kuijken, K., et al. 2013, The Messenger, 154, 32

Furusawa, H., Kosugi, G., Akiyama, M., et al. 2008, ApJS, 176, 1

Guzzo, L., Scodeggio, M., Garilli, B., et al. 2014, A\&A, 566, A108

Heymans, C., Van Waerbeke, L., Miller, L., et al. 2012, MNRAS, 427, 146

Huang, S., Leauthaud, A., Murata, R., et al. 2017, ArXiv eprints, arXiv:1705.01599

Hwang, H. S., Geller, M. J., Park, C., et al. 2016, ApJ, 818, 173

Ivezic, Z., Tyson, J. A., Abel, B., et al. 2008, ArXiv e-prints, arXiv:0805.2366

Iye, M., Ota, K., Kashikawa, N., et al. 2006, Nature, 443, 186

Jarvis, M. J., Bonfield, D. G., Bruce, V. A., et al. 2013, MNRAS, 428, 1281

Jurić, M., Kantor, J., Lim, K., et al. 2015, ArXiv e-prints, arXiv:1512.07914

Lawrence, A., Warren, S. J., Almaini, O., et al. 2007, MNRAS, 379,1599

Le Fèvre, O., Cassata, P., Cucciati, O., et al. 2013, A\&A, 559, A 14

LSST Science Collaboration, Abell, P. A., Allison, J., et al. 2009, ArXiv e-prints, arXiv:0912.0201

Mandelbaum, R., Miyatake, H., Hamana, T., et al. 2017, ArXiv e-prints, arXiv:1705.06745

Matsuoka, Y., Onoue, M., Kashikawa, N., et al. 2016, ApJ, 828, 26

Miyazaki, S., Komiyama, Y., Sekiguchi, M., et al. 2002, PASJ, 54, 833

Miyazaki, S., Oguri, M., Hamana, T., et al. 2015, ApJ, 807, 22

Newman, J. A., Cooper, M. C., Davis, M., et al. 2013, ApJS, 208, 5

Niemack, M. D., Ade, P. A. R., Aguirre, J., et al. 2010, in Proc. SPIE, Vol. 7741, Millimeter, Submillimeter, and Far-Infrared Detectors and Instrumentation for Astronomy V, 77411S 
Oguri, M., \& Takada, M. 2011, Phys. Rev. D, 83, 023008

Oguri, M., Lin, Y.-T., Lin, S.-C., et al. 2017a, ArXiv e-prints, arXiv:1701.00818

Oguri, M., Miyazaki, S., Hikage, C., et al. 2017b, ArXiv eprints, arXiv:1705.06792

Pierre, M., Pacaud, F., Adami, C., et al. 2016, A\&A, 592, A1

Schlegel, D. J., Finkbeiner, D. P., \& Davis, M. 1998, ApJ, 500, 525

Schneider, D. P., Gunn, J. E., \& Hoessel, J. G. 1983, ApJ, 264, 337

Steinhardt, C. L., Speagle, J. S., Capak, P., et al. 2014, ApJL, 791, L25

Swetz, D. S., Ade, P. A. R., Amiri, M., et al. 2011, ApJS, 194, 41

Takada, M., \& Jain, B. 2004, MNRAS, 348, 897

Takada, M., Ellis, R. S., Chiba, M., et al. 2014, PASJ, 66, R1

Tamura, N., Takato, N., Shimono, A., et al. 2016, in Proc. SPIE, Vol. 9908, Society of Photo-Optical Instrumentation Engineers (SPIE) Conference Series, 99081M

Tanaka, M., Coupon, J., Hsieh, B.-C., et al. 2017, ArXiv eprints, arXiv: 1704.05988

Thornton, R. J., Ade, P. A. R., Aiola, S., et al. 2016, ApJS, 227, 21

Toba, Y., Nagao, T., Strauss, M. A., et al. 2015, PASJ, 67, 86

van Haarlem, M. P., Wise, M. W., Gunst, A. W., et al. 2013, A\&A, 556, A2

Weinberg, D. H., Mortonson, M. J., Eisenstein, D. J., et al. 2013, Phys. Rep., 530, 87

York, D. G., Adelman, J., Anderson, Jr., J. E., et al. 2000, AJ, 120,1579 